468 DETERMINANTS OF SPORTS INJURY IN YOUNG FEMALE SWEDISH COMPETITIVE FIGURE SKATERS

${ }^{1}$ Moa Jederström, ${ }^{2}$ Sara Agnafors, ${ }^{3}$ Christina Ekegren, ${ }^{4}$ Kristina Fagher, 1,5 Håkan Gauffin, ${ }^{6}$ Laura Korhonen, ${ }^{7}$ Jennifer Park, ${ }^{1,8,9}$ Armin Spreco, ${ }^{1,8,9}$ Toomas Timpka. ${ }^{1}$ Athletics Research Center, Department of Health, Medicine and Caring Sciences, Linköping University, Linköping, Sweden; '2Department of Biomedical and Clinical Sciences, Division of Children's and Women's Health, Linköping University, Linköping, Sweden; ${ }^{3}$ Rehabilitation, Ageing and Independent Living (RAIL) Research Centre, School of Primary Allied Health Care, Monash University, Melbourne, Victoria, Australia; ${ }^{4}$ Department of Health Sciences, Rehabilitation Medicine Research Group, Lund University, Lund, Sweden; ${ }^{5}$ Department of Orthopedics and Department of Biomedical and Clinical Sciences, Linköping University, Linköping, Sweden; ${ }^{6}$ Department of Child and Adolescent Psychiatry and Department of Biomedical and Clinical Sciences, Center for Social and Affective Neuroscience, Linköping University, Linköping, Sweden; 'Institute of Clinical Sciences, Department of Surgery, Sahlgrenska Academy, University of Gothenburg, Gothenburg, Sweden; ${ }^{8}$ Department of Health, Medicine and Caring Sciences, Division of Society and Health, Linköping University, Linköping, Sweden; ${ }^{9}$ Center for Health Services Development, Region Östergötland, Linköping, Sweden

\subsection{6/bjsports-2021-IOC.429}

Background Although figure skating attracts several hundred thousand participants worldwide, there is little knowledge about physical health and sports injuries among young skaters. Objective To describe the health status of a geographicallydefined Swedish population of licensed competitive figure skaters and to examine injury determinants.

Design Cross-sectional.

Setting All licensed competitive skaters in the south-eastern region of Sweden.

Participants In total, 142 (36\%) of 400 skaters participated, 137 (96\%) of whom were girls (mean (SD) age: 12.9 (SD 3.0) years). Participating boys $(n=5)$ were excluded from further analysis.

Assessment of Risk Factors Age, skating level, eating habits, relative energy deficiency indicators, and training habits were assessed. Main Outcome Measurements The primary outcome measure was the one-year prevalence of a severe sports injury episode (time loss $>21$ days). The secondary outcome measure was the point prevalence of an ongoing sports injury.

Results The one-year prevalence of a severe sports injury episode was $31 \%$, which in the multiple model, was associated with older age (odds ratio (OR) 1.2, 95\% confidence interval (CI) $1.1-1.4 ; p=0.002)$ and an increased number of skipped meals per week (OR 1.1, 95\% CI 1.0-1.3; $\mathrm{p}=0.014)$. The point prevalence of an ongoing injury episode was 19\%, which was associated with older age (OR 1.4, 95\% CI 1.2$1.7 ; \mathrm{p}<0.001)$ and an increased number of skipped meals per week (OR 1.1, 95\% CI 1.0-1.3; p=0.049).

Conclusions One-third of young female Swedish competitive figure skaters had sustained a severe injury episode during the past year, and a fifth reported an ongoing episode. Older age and an increased number of skipped meals per week were associated with a sports injury episode. Further prospective studies of injury determinants among competitive figure skaters are warranted before the findings are broadly applied in intervention programs.

\section{RUNNING-RELATED INJURY IN COMPETITIVE ADOLESCENT DISTANCE RUNNERS: A QUALITATIVE STUDY OF PSYCHOSOCIAL RESPONSES}

${ }^{1}$ Robert Mann, ${ }^{2}$ Carly McKay, ${ }^{1}$ Alan Barker, ${ }^{1}$ Craig Williams, ${ }^{2}$ Bryan Clift. ${ }^{1}$ Children's Health and Exercise Research Centre, University of Exeter, Exeter, UK; ${ }^{2}$ Department for Health, University of Bath, Bath, UK

10.1136/bjsports-2021-IOC.430
Background Distance running is one of the most popular sports among children and adolescents around the world. Previous adult- and adolescent-based research indicates that injury is prevalent when participating in distance running. While knowledge related to the extent of the injury problem is important, an understanding of athletes' psychosocial responses to running-related injury (RRI), applying a qualitative lens of inquiry, is frequently overlooked.

Objective To investigate the psychosocial responses to 'serious RRI' (>28 days-6 months of time loss) in competitive adolescent distance runners in England.

Design Semi-structured interviews to facilitate a reflexive thematic analysis (deductive/latent) related to psychosocial responses to serious RRI.

Setting Competitive adolescent distance runners (i.e., $800 \mathrm{~m}$ to 10,000 m, including steeplechase) in England.

Patients (or Participants) Distance runners (13-18 y) were invited to participate if they had sustained a serious RRI within the previous 12-months, as self-reported via an online survey as part of a previous study. A total of 113 athletes completed the online survey, whereby 34 of these athletes had sustained at least one serious RRI.

Results Nineteen competitive adolescent distance runners were interviewed about their experiences of serious RRI, focussing on their response to and subsequent recovery from serious RRI. Based on a reflexive thematic analysis, three themes were developed: (1) performance uncertainty, (2) injury (mis) management, and (3) contested identity. These three themes were found to support a number of theoretical relationships proposed in Wiese-Bjornstal et al. (1998) integrated model of response to sport injury, alongside other previous research findings. In turn, each theme contributed towards an overarching understanding that serious RRI acts to destabilise the athletic identity of competitive adolescent distance runners, as a psychosocial recovery outcome.

Conclusions These findings will support the development of measures that aim to improve how competitive adolescent distance runners respond to RRI.

\section{PREVALENCE AND BURDEN OF HEALTH PROBLEMS IN MALE JUNIOR ELITE ICE HOCKEY PLAYERS - A 44- WEEK PROSPECTIVE COHORT STUDY}

${ }^{1,2}$ Anine Nordstrom, ${ }^{1}$ Roald Bahr, ${ }^{1,3}$ Ben Clarsen, ${ }^{2}$ Ove Talsnes. ${ }^{1}$ Department of Sports Medicine, Oslo Sports Trauma Research Center, NO, Oslo, Norway; ${ }^{2}$ Innlandet Hospital Trust, Elverum, Norway; ${ }^{3}$ Center for Disease Burden Norwegian Institute of Public Health, Bergen, No, Bergen, Norway

\subsection{6/bjsports-2021-IOC.431}

Background Little is known about the burden of overuse injuries and illnesses in junior elite ice hockey.

Objective The aim of this study was to describe the prevalence and burden of all health problems in junior elite ice hockey in Norway during one school year.

Design Prospective cohort study.

Setting Elite Sport Academy High Schools in Norway.

Participants 206 male junior ice hockey players.

Interventions (or Assessment of Risk Factors) Players reported all health problems, acute injuries, overuse injuries and illnesses weekly during the 2018/2019 school year (44 weeks) using the Oslo Sports Trauma Research Center Questionnaire on Health Problems. 
Main Outcome Measurements Prevalence and burden of acute injuries, overuse injuries and illnesses.

Results Of the players included, 25\% (95\% CI, 23-27\%) reported a health problem at any given time, while $16 \%$ (95\% CI, 15-17\%) experienced health problems with a substantial negative impact on training and performance. Of the total burden of health problems, acute injuries accounted for $42 \%$, overuse injuries $32 \%$ and illnesses $25 \%$. The greatest burden for acute injuries was caused by injuries to the hand, knee and ankle, whereas for overuse injuries the most burdensome location was the hip/groin and knee.

Conclusions This study documented that while acute injuries did represent the greatest problem among junior elite ice hockey players, overuse injuries, especially to the knee and hip/groin, also had substantial impact.

\section{Late Breaking Abstracts}

\section{THE FORCE EXPERIENCED BY THE HEAD DURING FOOTBALL HEADING IS MAINLY INFLUENCED BY THE SPEED OF THE BALL RATHER THAN ITS MECHANICAL PROPERTIES}

${ }^{1}$ Greg Tierney*, ${ }^{2}$ Jonathan Power, ${ }^{3}$ Ciaran Simms. ${ }^{1}$ School of Biomedical Sciences, University of Leeds, Leeds, UK; ${ }^{2}$ Football Association, London, UK; ${ }^{3}$ Trinity Centre for Biomedical Engineering, Trinity College Dublin, Dublin, Ireland

$10.1136 /$ bjsports-2021-IOC.432

Background There are growing concerns about the risk of neurodegenerative diseases associated with heading in football. It is essential to understand the biomechanics of football heading to guide player protection strategies to reduce the severity of the impact.

Objective The aim of this study was to assess the effect of football speed, mass and stiffness on the forces experienced during football heading using mathematical and human body computational model simulations.

Design Computational biomechanics study.

Setting N/A.

Patients (or Participants) N/A.

Interventions (or Assessment of Risk Factors) Football headers were reconstructed using a human body modelling approach. Simulations were run by independently varying the football mass, speed and stiffness.

Main Outcome Measurements Peak contact force experienced by the head was extracted from each simulation.

Results Mathematical and human body computational model simulations indicate that the force experienced by the head was directly proportional to the speed of the ball and directly proportional to the square root of the ball stiffness and mass. Conclusions Over the practical range of ball speed, mass and stiffness, the force experienced by the head during football heading is mainly influenced by the speed of the ball rather than its mass or stiffness. The findings suggest that it would be more beneficial to develop player protection strategies that aim to reduce the speed at which the ball is travelling when headed by a player. Law changes reducing high ball speeds could be trialled at certain age grades or as a phased introduction to football heading.

\section{BETWEEN-SIDE DIFFERENCES IN VELOCITY OF TRUNK ROTATIONS: CAN THIS IDENTIFY THE LIKELIHOOD OF LOW BACK PAIN?}

${ }^{1,2}$ Erika Zemkova* ${ }^{2}$ Michal Jelen. ${ }^{1}$ Department of Biological and Medical Sciences, Faculty of Physical Education and Sports, Comenius University, Bratislava, Slovakia; ${ }^{2}$ Sports Technology Institute, Faculty of Electrical Engineering and Information Technology, Slovak University of Technology, Bratislava, Slovakia

10.1136/bjsports-2021-IOC.433

Background A previous study [Zemková E et al. 2019] identified between-side differences in trunk rotational power in athletes trained in asymmetric sports. However, the question remains whether velocity of trunk rotations can also be considered specific to their asymmetric loading as one of the risk factors for low back disorders.

Objective This study compares velocity of trunk rotations on the dominant and non-dominant side in golfers, ice hockey players, tennis players, and age-matched controls.

Design Controlled laboratory study.

Setting Research laboratory.

Participants Groups of 17 golfers (age 23.3 \pm 3.1 y, height $177.3 \pm 8.1 \mathrm{~cm}$, body mass $85.6 \pm 10.7 \mathrm{~kg}), 17$ ice-hockey players (age 22.4 $\pm 2.7 \mathrm{y}$, height $182.6 \pm 7.3 \mathrm{~cm}$, body mass 88.0 $\pm 9.5 \mathrm{~kg}$ ), 21 tennis players (age $20.9 \pm 2.9 \mathrm{y}$, height 179.9 $\pm 5.9 \mathrm{~cm}$, body mass $81.0 \pm 8.6 \mathrm{~kg}$ ), and 39 controls (age 21.8 $\pm 2.4 \mathrm{y}$, height $177.6 \pm 7.4 \mathrm{~cm}$, body mass $79.6 \pm 11.1 \mathrm{~kg}$ ) participated in the study.

Assessment Basic biomechanical parameters during trunk rotations with different weights $(1,5.5,10.5,15.5$ and $20 \mathrm{~kg}$ ) were measured using the FiTRO Torso Premium.

Main Outcome Measurements Peak velocity and mean velocity in the acceleration phase of trunk rotations.

Results Mean velocity of trunk rotations was significantly higher on the dominant than non-dominant side at weights of $1 \mathrm{~kg} \quad(11.9 \%, \mathrm{p}=0.033), \quad 5.5 \mathrm{~kg} \quad(13.3 \%, \mathrm{p}=0.025), \quad 10.5 \mathrm{~kg}$ (13.7\%, p=0.021), $15.5 \mathrm{~kg} \quad(14.1 \%, \quad \mathrm{p}=0.017)$, and $20 \mathrm{~kg}$ $(15.4 \%, \mathrm{p}=0.011)$ in ice-hockey players, at $1 \mathrm{~kg}(11.1 \%$, $\mathrm{p}=0.043), \quad 5.5 \mathrm{~kg} \quad(11.5 \%, \quad \mathrm{p}=0.039), \quad 10.5 \mathrm{~kg} \quad(12.9 \%$, $\mathrm{p}=0.030)$, and $15.5 \mathrm{~kg}(13.6 \%, \mathrm{p}=0.025)$ in tennis players, and at $1 \mathrm{~kg}(15.4 \%, \mathrm{p}=0.015), 5.5 \mathrm{~kg}(16.5 \%, \mathrm{p}=0.009)$ and $10.5 \mathrm{~kg}(16.6 \%, \mathrm{p}=0.008)$ in golfers. However, its values at these weights did not differ significantly $(<10 \%)$ in the control group. Similar trend was observed for peak velocity of trunk rotations.

Conclusions Though significant between-side differences in trunk rotational velocity in athletes trained in asymmetric sports may help to identify those at risk of a low back pain, a causal relationship between these factors have yet to be investigated. 